\title{
Pancreatic carcinoma presenting as bleeding from segmental gastric varices: pitfalls in diagnosis
}

\author{
F.J. Mullan and S.T.D. McKelvey
}

Surgical Department, The Ulster Hospital, Dundonald, Belfast BT16 0RH, Northern Ireland.

\begin{abstract}
Summary: Splenic vein occlusion leading to gastric variceal haemorrhage should be considered in cases of obscure upper gastrointestinal bleeding. We report an unusual case in which the underlying pathology was a resectable carcinoma of the pancreatic tail.
\end{abstract}

\section{Introduction}

Segmental gastric varices due to isolated splenic vein thrombosis is an unusual complication of pancreatic pathology. Early diagnosis is rarely made but may be vital where the underlying pathology is malignant. We describe a case of operable pancreatic carcinoma presenting with bleeding gastric varices which illustrates the diagnostic pitfalls and the need for a better awareness of the syndrome.

\section{Case report}

A 63 year old woman with maturity onset diabetes was admitted following a haematemesis and melaena. Urgent barium meal examination suggested carcinoma of the cardia of the stomach. A further massive haematemesis prompted emergency surgery. At laparotomy there was no evidence of tumour. The gastric mucosa was friable and bled easily on contact. Several areas were undersewn. Truncal vagotomy and pyloroplasty were performed. The spleen was noted to be slightly enlarged. Six months after initial presentation the patient was readmitted with recurrent anaemia. Repeat barium meal again showed fundal mucosal distortion (Figure 1a) indistinguishable from malignant infiltration. Oesophagogastro-duodenoscopy failed to establish any site of mucosal destruction and the fundus appeared normally distensible.

At laparotomy the spleen was twice normal size. There was no infiltration or ulceration of the gastric fundus but the gastroepiploic and short gastric veins were markedly distended. The lesser sac was opened and after thorough exploration a small firm tumour was found in the pancreatic tail encroaching upon and occluding the splenic vein.

Correspondence: F.J. Mullan, F.R.C.S.

Accepted: 12 May 1989
Splenectomy and distal pancreatectomy were performed. Histology confirmed a $3.5 \times 2 \mathrm{~cm}$ adenocarcinoma of the pancreatic tail infiltrating around and compressing large blood vessels. Barium meal performed five months postoperatively showed complete resolution of the fundal varices (Figure 1b) and ultrasonic scanning confirmed patency of the portal vein.

\section{Discussion}

The splenic vein running along the postero-superior aspect of the pancreas is vulnerable to compression or infiltration by adjacent pancreatic lesions. This results in isolated splenic vein thrombosis (ISVT) and an unusual form of extrahepatic portal hypertension. An extensive collateral circulation develops involving the segment of stomach drained by the short gastric veins (Figure 2). Bleeding may occur from the resultant gastric varices.

Sutton $^{1}$ and Madsen ${ }^{2}$ in comprehensive reviews of English language publications from 1900-69 and 1969-84, respectively, identified only 263 cases. Almost half the cases were secondary to pancreatitis or its sequelae, notably pseudocyst formation. Only 36 cases were attributable to adenocarcinoma of the pancreas, the vast majority of which were irresectable.

Diagnostic delay is common. In Madsen's series only $49 \%$ of patients were correctly diagnosed at the time of first bleed, the remainder having a median diagnostic delay of 11 months. The correct diagnosis was missed at first operation in $22 \%$ of patients undergoing laparotomy for gastrointestinal bleeding. Only $43 \%$ of barium studies correctly diagnosed gastric varices ${ }^{3}$ which were completely missed in $29 \%$ of cases. Endoscopy was no more sensitive with localized gastric varices correctly diagnosed in less than $40 \%$ of cases. ${ }^{3,4}$ Experience of ISVT outside major referral centres is limited. 
a

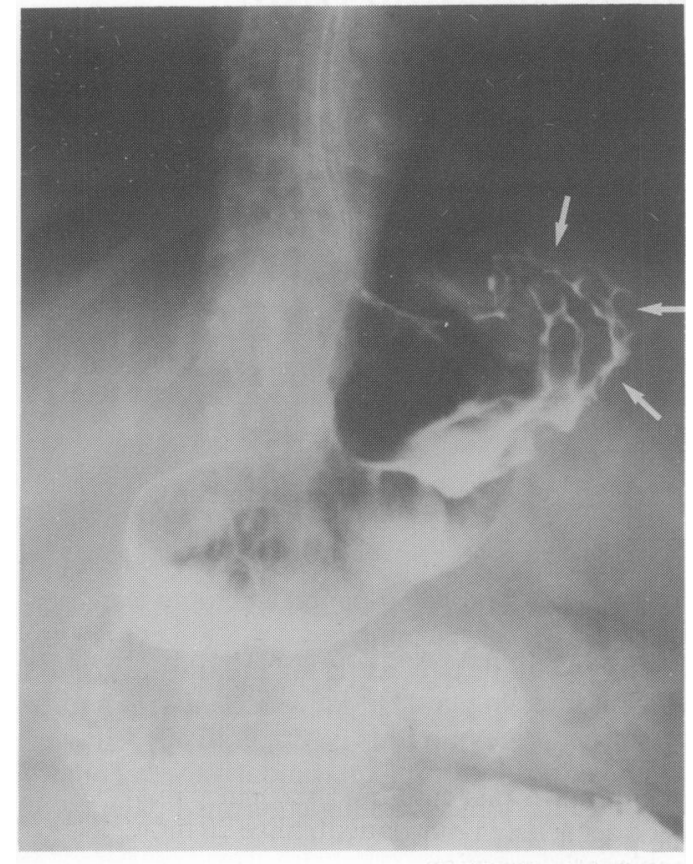

b

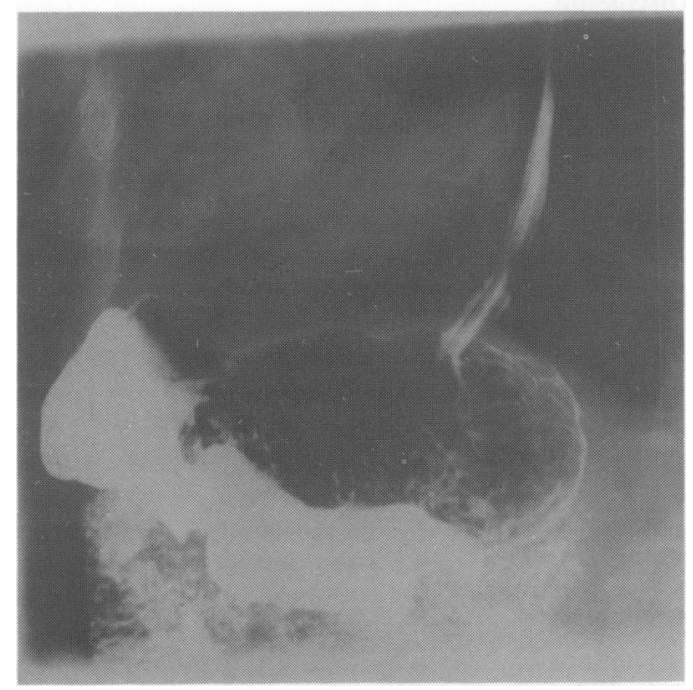

Figure 1 (a) Barium meal demonstrating gastric varices as prominent irregular mucosal folds (arrows) limited to the gastric fundus. (b) Resolution of varices after splenectomy and resection of pancreatic tumour.

What then are the features which might alert the gastroenterologist, radiologist, endoscopist or surgeon to the possibility of this diagnosis?

The key to early diagnosis is a high index of suspicion. Symptoms may be limited to those of the underlying disease. The two commonest clinical features, gastrointestinal blood loss and splenomegaly, ${ }^{5}$ are particularly suspicious when associated with a history of pancreatitis or recurrent upper abdominal pain. Bleeding is frequently severe enough to cause haematemesis ${ }^{2}$ but the source may have remained obscure following previous investigations. ${ }^{6}$ Splenic enlargement is common but not invariable ${ }^{7-9}$ and may only be apparent at laparotomy. Clinical splenomegaly is apparent in about half of reported cases. ${ }^{1-3}$ Evidence of hypersplenism ${ }^{10}$ is diagnostically helpful but infrequently found ${ }^{8}$ Liver function tests are invariably normal unless there is metastatic liver disease or alcohol abuse.

Gastric varices appear on barium studies as thick distorted, tortuous mucosal folds or filling defects over the greater curvature of the stomach extending towards the cardia. ${ }^{11}$ Confusion with gastric neoplasm is commonplace. ${ }^{12}$ Co-existing splenomegaly is more suggestive of varices. Isolated gastric varices must be included in the differential diagnosis of any polypoid lesion or hypertrophic rugae localized to the gastric fundus.

Ultrasonography is a useful non-invasive method of confirming patency of the portal vein and with computed tomographic scanning may help to localize an underlying pancreatic mass. The position and patency of the splenic vein itself are more difficult to identify by ultrasonography.

ISVT may be confirmed pre-operatively by splenoportography or the venous phase of selective angiography. The latter procedure is now considered the method of choice being safer and more easily controlled than percutaneous splenoportography. ${ }^{3}$

Endoscopic misinterpretation or hazardous, even lethal, ${ }^{13}$ biopsy may result unless ISVT is considered when a nodular submucosal mass or prominent fundal folds are found in the stomach. ${ }^{4}$ Suspicion of ISVT should be high when a local fundal abnormality identified radiologically is not confirmed endoscopically. Oesophageal varices are normally absent unless the short gastric collateral system is inadequate to decompress the splenic axis or the coronary vein inserts into the splenic vein rather than the portal vein..$^{14}$

Dilated gastroepiploic veins, particularly in the presence of a large spleen and a normal liver, are very suggestive of ISVT. These may be less obvious in a hypotensive patient when emergency laparotomy is undertaken for profound haematemesis. Gastric varices should be considered when laparotomy fails to reveal a convincing site of haemorrhage in a blood filled stomach. ${ }^{15,16}$

Bleeding from gastric varices due to benign ISVT is almost always cured by splenectomy. Malignant ISVT usually implies extrapancreatic extension and a very poor prognosis..$^{6,8,13,17-19}$ Several pan- 


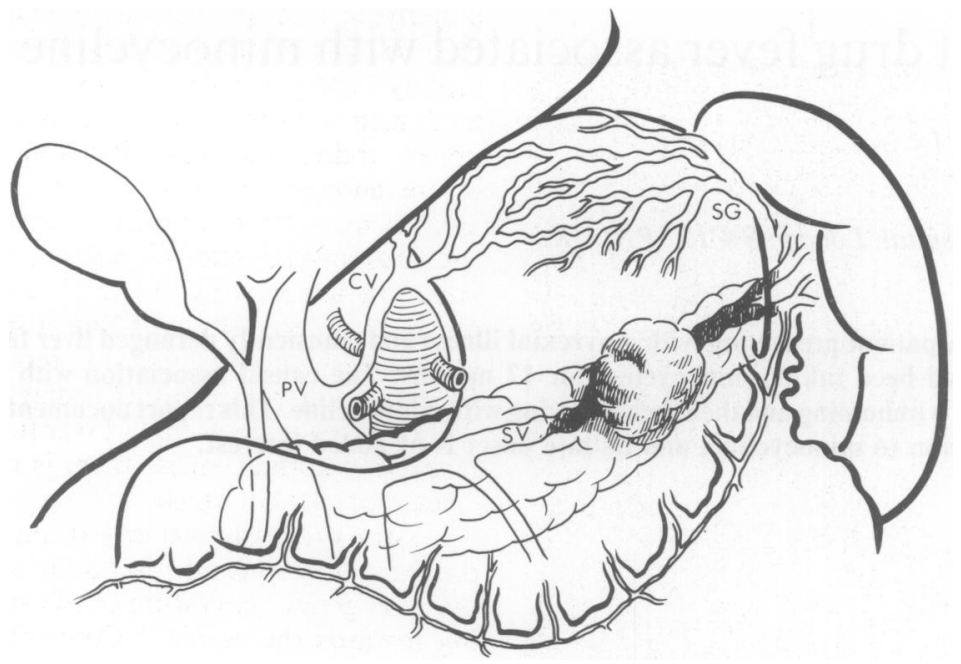

Figure 2 Pancreatic tumour causing isolated splenic vein thrombosis. Occlusion of the splenic vein (SV) leads to collateral venous drainage via the short gastric (SG) and coronary veins (CV) to the patent portal vein (PV).

creatic carcinomas causing ISVT have been resected ${ }^{10,16}$ and Hurwitt ${ }^{16}$ reported a case surviving 19 months postoperatively. In view of the pathological findings we could not claim a curative operation in this case but excellent palliation has been achieved in our patient, alive 20 months after pancreatic resection.

\section{References}

1. Sutton, J.P., Yarborough, D.Y. \& Richards, J.T. Isolated splenic vein occlusion. Arch Surg 1970, 100: 623-626.

2. Madsen, M.S., Petersen, T.H. \& Sommer, H. Segmental portal hypertension. Ann Surg 1986, 204: 72-77.

3. Moossa, A.R. \& Gadd, M.A. Isolated splenic vein thrombosis. World J Surg 1985, 9: 384-390.

4. Bachman, B.A. \& Brady, P.G. Localised gastric varices: mimicry leading to endoscopic misinterpretation. Gastro Endoscopy 1984, 30: 244-247.

5. Glynn, M.J. Isolated splenic vein thrombosis. Arch Surg 1986, 121: 723-725.

6. Madsen, M.S. Splenic vein obstruction: a cause of upper gastrointestinal bleeding. Acta Chir Scand 1986, 152: 393-395.

7. Itzchak, Y. \& Glickman, M.G. Splenic vein thrombosis in patients with a normal size spleen. Invest Radiol 1977, 12: 158-163.

8. Johnston, F.R. \& Myers, R.T. Etiological factors and consequences of splenic vein obstruction. Ann Surg 1973, 177: 736-739.

9. Muhletaler, C., Gerlock, A.J., Goncharenko, V., Avant, G.R. \& Flexner, J.M. Gastric varices secondary to splenic vein occlusion: radiographic diagnosis and clinical significance. Radiology 1979, 132: 593-598.

10. Marks, L.J., Weingarten, B. \& Gerst, G.R. Carcinoma of the tail of the pancreas associated with bleeding gastric varices and hypersplenism. Ann Intern Med 1952, 37: 1077-1084.
11. Marshall, J.P. II, Smith, P.D. \& Hoyumpa, A.M. Jr. Gastric varices: problem in diagnosis. Am J Dig Dis 1977, 22: 947-955.

12. Karr, S. \& Wohl, G.T. Clinical importance of gastric varices. $N$ Engl J Med 1960, 263: 665-669.

13. Rosch, W. Isolated gastric varices - a hint of pancreatic disorders. Endoscopy 1974, 6: 217-220.

14. Little, A.G. \& Moossa, A.R. Gastrointestinal haemorrhage from left-sided portal hypertension. Am J Surg 1981, 141: 153-158.

15. Salam, A.A., Warren, W.D. \& Tyras, D.H. Splenic vein thrombosis: a diagnosable and curable form of portal hypertension. Surgery 1973, 74: 961-972.

16. Hurwitt, E.S., Altman, S.F., Gerst, G.R. \& Webber, B.M. Gastrointestinal bleeding due to splenic vein obstruction by pancreatic tumours. Arch Surg 1954, 68: 7-11.

17. Manenti, A., Splenic vein obstruction secondary to pancreatic carcinoma. Acta Chir Belg 1981, 80: 245-248.

18. Keith, R.G., Mustard, R.A. \& Saibil, E.A. Gastric variceal bleeding due to occlusion of splenic vein in pancreatic disease. Can J Surg 1982, 25: 301-304.

19. Alberti-Flor, J.J., Evans, J. \& Dunn, G.D. Pancreatic carcinoma presenting with isolated bleeding gastric varices. $J$ Tenn Med Assoc 1985, 78: 81-82. 